\title{
Nusinersen for SMA: expanded access programme
}

\author{
Michelle A Farrar, ${ }^{1,2}$ Hooi Ling Teoh, ${ }^{1,2}$ Kate A Carey, ${ }^{1,2}$ Anita Cairns, ${ }^{3}$ Robin Forbes, ${ }^{4,5}$ \\ Karen Herbert, ${ }^{6}$ Sandra Holland, ${ }^{1}$ Kristi J Jones, ${ }^{1,7}$ Manoj P Menezes, ${ }^{1,7}$ \\ Margot Morrison, ${ }^{1}$ Kate Munro, ${ }^{3}$ Daniella Villano, ${ }^{4}$ Richard Webster, ${ }_{1}^{1,7}$ \\ Ian R Woodcock, ${ }^{4,5}$ Eppie M Yiu, ${ }^{4,5}$ Hugo Sampaio, ${ }^{1,2}$ Monique M Ryan ${ }^{4,5,8}$
}

\begin{abstract}
'Department of Neurology, Sydney Children's Hospitals Network, Sydney, Australia ${ }^{2}$ School of Women's and Children's Health, UNSW Medicine, The University of New South Wales, Sydney, Australia ${ }^{3}$ Department of Neurology, Lady Cilento Children's Hospital, Brisbane, Australia ${ }^{4}$ Department of Neurology, The Royal Children's Hospital, Melbourne, Australia

${ }^{5}$ Murdoch Children's Research Institute, Melbourne, Australia ${ }^{6}$ Department of Physiotherapy, Sydney Children's Hospitals Network, Sydney, Australia ${ }^{7}$ Discipline of Paediatrics and Child Health, The Children's Hospital at Westmead Clinical School, The University of Sydney, Sydney, Australia ${ }^{8}$ Department of Paediatrics, The University of Melbourne, Melbourne, Australia
\end{abstract}

\section{Correspondence to}

Dr Michelle A Farrar, School of Women's and Children's Health and Sydney Children's Hospital, High Street Randwick, NSW 2031, Australia; m.farrar@unsw. edu.au

Received 9 October 2017 Revised 23 January 2018 Accepted 4 February 2018 Published Online First 16 March 2018

\section{ABSTRACT}

Background Spinal muscular atrophy (SMA) is a devastating motor neuron disorder causing progressive muscle weakness and respiratory insufficiency. We present the initial Australian experiences implementing the expanded access programme (EAP) to enable preapproval access to nusinersen, the first diseasemodifying therapy, for SMA type 1.

Methods An Australian multicentre, open-label EAP for nusinersen enrolled patients with infantile-onset SMA type 1 from November 2016 to September 2017. Standard-of-care medical therapy and treatment with intrathecal nusinersen were provided to all patients. Clinical and diagnostic characteristics, molecular genetics, treatment administered, and functional motor outcomes were assessed.

Results A total of 20 patients with SMA type 1 met the inclusion criteria, of whom 16 consented and received nusinersen treatment. Median time to diagnosis from symptom onset was 5.0 months and was correlated with age of onset $(r=0.54, P<0.05)$. Management shifts included proactive nutritional and pulmonary support in all newly diagnosed patients with increased complexity of decision making. Supplemental nutrition with or without nocturnal non-invasive ventilation was implemented during follow-up in new diagnoses with age of onset $<3$ months and 2 SMN2 copies.

Conclusions The nusinersen EAP highlights difficulties in achieving early diagnosis and/or prevention, the evolution of optimal clinical care in a time of uncertain prognostication, resource implications and ethical issues in clinical practice for SMA type 1. These challenges are broadly relevant to the realisation of all novel therapeutics in neurological disorders.

\section{INTRODUCTION}

Spinal muscular atrophy (SMA) is a devastating motor neuron disorder causing progressive muscle weakness and respiratory insufficiency, often resulting in early death. Approximately 1 in 11000 individuals are affected, $60 \%$ of whom have the most severe form, SMA type 1, with onset of profound weakness and hypotonia by 6 months of age, and failure to ever achieve independent sitting. ${ }^{1}$ SMA is the leading genetic cause of infant mortality. The most common cause of SMA is mutations in the survival motor neuron 1 (SMN1) gene, which result in SMN protein deficiency. ${ }^{2}$ Until recently there has been no disease-modifying treatment, and the management of
SMA focused on managing the complications caused by weakness, feeding and breathing difficulties. ${ }^{3}$

Recent clinical trials of nusinersen, an antisense oligonucleotide developed to alter the splicing of SMN2 pre-mRNA and thus increase concentrations of functional SMN protein, have shown acceptable safety and tolerability and promising clinical efficacy. ${ }^{4-6}$ Nusinersen is administered intrathecally, most often with four loading doses over an 8-week period, followed by 4-monthly maintenance therapy. Following expedited regulatory review, nusinersen has been approved as the first pharmacological treatment for SMA in the USA, Europe, Canada and Japan. Concomitantly, an expanded access programme (EAP) to provide nusinersen to patients with SMA type 1 commenced in Australia while applications for accelerated regulatory review and reimbursement approval were undertaken in this country. For the first time, management of SMA type 1 in Australia can include disease-specific active therapy in addition to multidisciplinary supportive care. This has changed prognosis and life expectancy in infants receiving nusinersen. Here we describe initial Australian experiences in implementing nusinersen for patients with SMA type 1 in a clinical setting, the accompanying paradigm shifts in management, and the practical and ethical challenges as SMA enters a new treatment era.

\section{METHODS}

This was a prospective multicentre study of patients with SMA type 1 who attended neuromuscular clinics at the Sydney Children's Hospitals Network, Lady Cilento Children's Hospital Brisbane or Royal Children's Hospital Melbourne for clinical care during the first 10 months of an EAP to provide nusinersen to patients with SMA type 1 in Australia, from November 2016 to September 2017. The EAP enabled patients with SMA type 1 to access nusinersen free of charge to the patient, hospital or state before the full process of regulatory approval in Australia is completed. Informed consent for treatment was obtained from all cases.

The inclusion criteria were confirmed homozygous SMN1 deletions or compound heterozygous SMN1 mutations, onset of clinical signs and symptoms before 6 months of age, and inability to sit independently, consistent with infantile-onset (type I) SMA. Patients participating in a programme with an investigational therapy for SMA within 6 months were excluded from the present study. 
Medical therapy conformed to the 2007 Consensus Statement for Standard of Care in SMA. ${ }^{3}$

Participants received intrathecal age-adjusted doses of nusinersen $(2.4 \mathrm{mg} / \mathrm{mL})$ proportional to their cerebrospinal fluid (CSF) volume via lumbar puncture, with loading doses on days 1, 15, 30 and 60 , followed by 4-monthly maintenance doses in line with manufacturer guidelines. Approximately 3-5 mL of CSF was collected prior to dose administration. Injections were given over 1-2 min via a 22-gauge or 24-gauge needle. Topical local anaesthesia was used without sedation in infants younger than 12 months. Older patients variably received only topical anaesthesia, conscious sedation with nitrous oxide or oral midazolam, or a brief general anaesthetic using propofol or volatile agents.

The following clinical data were recorded: age of onset, age of diagnosis, consultations preceding diagnosis, SMN2 gene copy number and management parameters. SMA type 1 was further subclassified based on age of onset. ${ }^{7}$ Neonates with SMA type $1 \mathrm{a}$ are severely affected at birth with joint contractures, paucity of movement and often require ventilatory support. Infants with SMA type $1 \mathrm{~b}$ have onset by the age of 3 months, paradoxical breathing and a bell-shaped chest. Infants with SMA type 1c have onset between 3 and 6 months of age, may have paradoxical breathing, a bell-shaped chest and some head control. Event-free survival (ventilator support $>16$ hours/day or death) and change from baseline of the Hammersmith Infant Neurological Exam motor milestones portion-part 2 (HINE-2), ${ }^{8}$ a general categorical measure of motor milestone development for infants, assessed clinical response to provide context for clinical care. Domains included ability to kick (supine), head control, rolling, sitting, crawling, standing and walking. Clinical care and decision making during the EAP were recorded.

Descriptive statistics were used to summarise clinical features (number of patients, mean, median with range). Categorical outcomes were summarised with frequencies and percentages. Correlations between age of onset and time to diagnosis in patients with homozygous SMN1 deletions were analysed by Spearman's rank correlation coefficient. A probability $(\mathrm{P})$ value of $<0.05$ was considered statistically significant. We included all patients who received at least one dose of nusinersen in our analysis.

\section{RESULTS}

\section{Patient characteristics and diagnosis of SMA}

A total of 20 patients met the inclusion criteria, with 16 consenting and receiving nusinersen treatment, of whom 8 were new presentations and 8 were diagnosed preceding the initiation of the nusinersen EAP. Four families chose palliative care to optimise quality of life: reasoning included considering treatment with nusinersen a non-curative, life-prolonging measure, and feeling that nusinersen treatment was overly burdensome, while in two cases disease progression was so advanced that the family felt that it was not in their child's best interests to subject them to intrathecal treatments of uncertain long-term benefit.

The baseline characteristics of the cohort are summarised in table 1. Antenatal history determined that 15 of $16(94 \%)$ pregnancies were conceived naturally; 3 followed multiple unsuccessful cycles of in vitro fertilisation (IVF). One child had a family history of SMA. All families were unaware of the availability of preconception carrier screening for SMA with the option of prenatal or preimplantation genetic diagnosis. Nineteen of 20 (95\%) had homozygous SMN1 deletions and 1/20 (5\%) had compound heterozygous SMN1 mutations. The median disease duration at time of diagnosis (time from
Table 1 Baseline demographics and clinical characteristics of Australian patients with infantile-onset SMA type 1 enrolled in the nusinersen $\operatorname{EAP}(n=16)$

\begin{tabular}{lll}
\hline & $\begin{array}{l}\text { New SMA diagnosis } \\
\text { during nusinersen EAP, } \\
\mathbf{n}=8(\%)\end{array}$ & $\begin{array}{l}\text { SMA diagnosis } \\
\text { preceding initiation of } \\
\text { nusinersen EAP, } \mathbf{n}=8 \text { (\%) }\end{array}$ \\
\hline $\begin{array}{l}\text { Gender } \\
\text { Male }\end{array}$ & 5 \\
\hline Female & 3 & 3 \\
\hline SMN2 copy number & & 5 \\
\hline 2 copies SMN2 & 4 & $2^{*}$ \\
\hline 3 copies SMN2 & 4 & 4 \\
\hline $\begin{array}{l}\text { Age of symptom onset } \\
\text { (months) }\end{array}$ & & \\
\hline $\begin{array}{l}\text { Median } \\
\text { Range }\end{array}$ & 2.8 & 5.1 \\
\hline $\begin{array}{l}\text { Age at diagnosis (months) } \\
\text { Median }\end{array}$ & $1-5$ & $3-5.9$ \\
\hline Range & 6.4 & 10.5 \\
\hline $\begin{array}{l}\text { Age at initiation of } \\
\text { nusinersen (months) }\end{array}$ & $2.1-11$ & $7-72 \dagger$ \\
\hline Median & 7.5 & 102.4 \\
\hline Range & $2.5-11.9$ & $27.9-433.8$ \\
\hline
\end{tabular}

*SMN2 copy number not available in two patients.

†The patient with compound heterozygous SMN1 mutations was diagnosed at aged 6 years following second opinion from neuromuscular expert. Review of initial genetic testing prompted SMN1 sequencing studies.

EAP, expanded access programme; SMA, spinal muscular atrophy.

onset of symptoms to diagnosis) was 5.0 months (range $0.5-72$ months). Variation in time to diagnosis was significantly associated with age of onset $(\mathrm{r}=0.54, \mathrm{P}<0.05$; figure 1$)$. Maternal child nurses, general practitioners, physiotherapists and paediatricians were important in establishing the diagnosis of SMA, with parental concerns regarding low tone and/or motor development being raised during consultations with these healthcare professionals. The approach to these parental concerns varied individually and within each craft group, ranging from dismissal, monitoring of further development or non-urgent paediatric/ neurology referral, to immediate SMN1 gene testing and urgent referral to a paediatric neurologist.

A spectrum of severity was evident; specifically, 4/8 (50\%) new diagnoses had SMA type 1b, and 4/4 (100\%) of these had 2 SMN2 copies. In patients diagnosed with SMA type 1c, 8/12 had 3 SMN2 copies, 2/12 had 2 copies and 2/12 had unknown SMN2 copies.

Following diagnosis, paediatric subjects were followed in a multidisciplinary neuromuscular clinic staffed by neurologists, specialty nurses, respiratory physicians, orthopaedic surgeons, genetic counsellors, physiotherapists and other allied health personnel. All received treatments as per established standards of care for SMA1. ${ }^{3}$ The adult patients had neurologists and respiratory physicians but did not have access to a multidisciplinary adult neuromuscular clinic. One patient had previously received hydroxybutyrate as part of a USA-based clinical trial and three others had previously trialled sodium valproate. Significant history included surgical repair of coarctation of the aorta at 6 weeks of age in one patient. Three male patients had undescended testes.

At the time of initiation of nusinersen, 8/8 newly diagnosed subjects were entirely orally fed and ventilating unaided. Among patients diagnosed preceding the initiation of the nusinersen EAP, $1 / 8$ patients received feeds via a nasogastric tube, and 5/8 


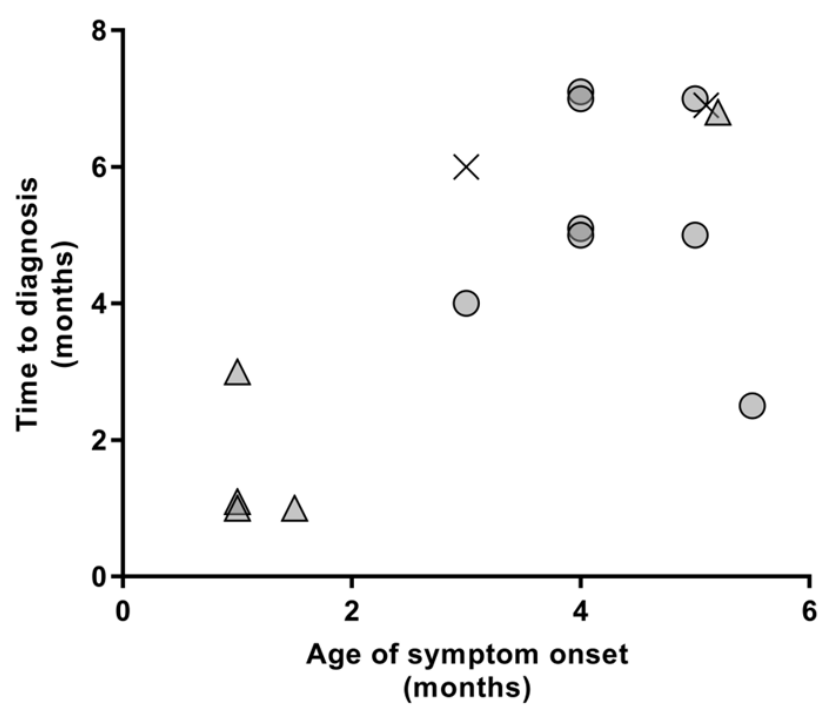

Figure 1 Relationship between age of onset and disease duration at diagnosis of SMA type 1 in patients with homozygous SMN1 deletions. Two SMN2 copies (triangles), 3 SMN2 copies (circles) and unknown SMN2 copy number (crosses) are plotted. SMA, spinal muscular atrophy.

were fed via a gastrostomy tube (the average age of commencing enteral feeds was 9.2 years). One of the eight patients had undergone spinal fusion, at age 12 years, and seven received nocturnal non-invasive ventilatory support (the average age of commencing nocturnal non-invasive ventilation was 6.4 years). Consistent with standard Australian practice, none had a tracheostomy.

\section{Decision making and consent}

Concepts discussed during the informed consent process included the current state of knowledge regarding the clinical efficacy, safety and side effects of nusinersen, based on recent publications and conference presentations, and the uncertainties regarding the short-term and long-term impact of this therapy on disease progression in SMA. Specific points covered included that nusinersen may modify disease severity and necessitate ongoing medical intervention (intrathecal injections); that patients do not uniformly have a promising clinical response (survival and motor function); and there was a likelihood of significant chronic physical disability in children treated with nusinersen. The therapeutic goals varied among patients with SMA type 1 and their families, with enthusiasm to pursue active therapy and supportive care in 14/16, while 2/16 had initial hesitation and concern related to long-term disability. In patients with SMA type 1 diagnosed before the availability of nusinersen, therapeutic goals encompassed improvement of quality of life, particularly respiratory function, and maintaining or improving motor function. Older patients often focused on specific aspects of their physical function which they wished to retain or even improve, such as their ability to actively extend their wrists (for typing and texting), to flex their elbows (to self-feed), and their ability to speak clearly and manage their oral secretions.

\section{Effects on clinical care}

The management of patients is summarised in table 2. The median patient age at commencement of nusinersen was 20.0 months (range 2.5 months-35 years). Lumbar punctures were successfully performed in all patients. CT guided the procedures in an adolescent with spinal fusion (figure 2). At the time of clinical censoring, the median duration of nusinersen treatment was 5.1 months (1.2-10.7 months); 16/16 were alive and not using ventilation $>16$ hours/day and $0 / 16$ had discontinued nusinersen. All patients had some improvement or stabilisation in motor milestones after day 60.

Optimal management of nutrition was a therapeutic goal in all patients, with universal dietitian assessment and management as part of multidisciplinary care, which also included speech pathology assessment when clinically indicated. Three of four recently diagnosed patients with symptom onset before 3 months and 2 SMN2 copies required supplemental enteral nutrition via nasogastric tube or gastrostomy during clinical follow-up. In contrast, all newly diagnosed patients with symptom onset after 3 months and 3 SMN2 copies $(n=4)$ remained orally fed during clinical follow-up. Gastrostomy insertion was undertaken in consultation with surgical, anaesthetic, intensive care and respiratory departments.

Philosophical and ethical discussions regarding acute and chronic respiratory management of SMA type 1 were undertaken with all families shortly after diagnosis. Given the uncertainties regarding individual response to treatment, definitive care plans were generally not made, although there was universal agreement to treat reversible respiratory illnesses. Respiratory care included teaching of airway clearance techniques and assisted coughing. Shortly following commencement of nusinersen, newly diagnosed patients were referred for overnight polysomnography to guide considerations of non-invasive ventilation. Two of four newly diagnosed patients with SMA type $1 \mathrm{~b}$ had abnormal polysomnograms; one commenced nocturnal non-invasive ventilation at age 4 months. Polysomnograms were normal in three of four patients with SMA type 1c (one is pending); none commenced nocturnal non-invasive ventilation during clinical follow-up. Prophylactic non-invasive ventilation was not initiated on any patient.

New rehabilitative approaches became evident over the period of the EAP, corresponding with shifts in parental goals from palliative comfort and optimal positioning to active therapy to prevent contractures and promote mobility. These new approaches included stretches, provision of splints, standing frames and orthotics (including thoracolumbar orthoses for scoliosis) and mobility equipment, for example Wizzybugs and power wheelchair planning. In two patients surveillance hip X-ray screening demonstrated bilateral hip subluxation. In the first case management consisted of a hip orthosis and pending bilateral rotational osteotomy, while the second was urgently referred for orthopaedic intervention in anticipation of the patient achieving weight bearing.

Different approaches to comorbidities were also evident. Surgical referral and planning for orchidopexy for undescended testes were undertaken. The infant with previous aortic coarctation presented acutely with 3 days of reduced feeding, tachypnoea, respiratory distress, sweating with feeding and lethargy. Investigations determined recurrence of aortic narrowing, prompting aortic balloon angioplasty with resolution of acute symptoms (figure 2). Clinical psychology support for grief counselling, depression and decision making was provided to all families at the time of diagnosis, and subsequently as required.

\section{DISCUSSION}

The natural history and treatment of SMA have been transformed by availability of nusinersen, the first agent shown to ameliorate, and in some cases reverse, progression of muscle weakness in this disorder. We describe Australian experiences implementing nusinersen for patients with SMA type 1 in a 


\begin{tabular}{|c|c|c|c|c|}
\hline $\begin{array}{l}\text { Patient (SMN2 copy } \\
\text { number) }\end{array}$ & $\begin{array}{l}\text { Age at nusinersen } \\
\text { initiation }\end{array}$ & Nutritional management & Pulmonary management & $\begin{array}{l}\text { Motor milestone response* (duration } 0 \\
\text { nusinersen) }\end{array}$ \\
\hline \multicolumn{5}{|l|}{ Newly diagnosed } \\
\hline \multicolumn{5}{|l|}{ SMA 1b } \\
\hline $1(2)$ & 2.5 months & Entirely oral fed & $\begin{array}{l}\text { Sleep-disordered breathing at age } \\
2 \text { months, repeat study planned to } \\
\text { consider initiation of NIV }\end{array}$ & Not reported (1.2 months) \\
\hline $2(2)$ & 2.7 months & $\begin{array}{l}\text { Gastrostomy, enteral feeds from age } \\
4 \text { months }\end{array}$ & $\begin{array}{l}\text { NIV from } 4 \text { months for sleep-disordered } \\
\text { breathing, hospital admission for } \\
\text { aspiration pneumonia-NIV+O }{ }_{2}\end{array}$ & Improved head control (5.0 months) \\
\hline $3(2)$ & 2.9 months & $\begin{array}{l}\text { Enteral feeds only when unwell, } \\
\text { gastrostomy }\end{array}$ & Normal PSG at age 6 months & $\begin{array}{l}\text { Kicking-horizontal, full upright head control } \\
\text { (5.1 months) }\end{array}$ \\
\hline $4(2)$ & 4.2 months & $\begin{array}{l}\text { Gastrostomy inserted, enteral } \\
\text { supplementation from age } 9 \text { months }\end{array}$ & Normal PSG at age 14 months & $\begin{array}{l}\text { Kicking-touches toes, full upright head } \\
\text { control, rolls to side, stable sitting } \\
\text { (10.7 months) }\end{array}$ \\
\hline \multicolumn{5}{|l|}{ SMA 1C } \\
\hline $5(3)$ & 10.7 months & Entirely oral fed & Normal PSG & Kicks legs horizontally ( 2.4 months) \\
\hline $6(3)$ & 10.9 months & Entirely oral fed & Normal PSG at age 11 months & Sitting with support at hips (6.8 months) \\
\hline $7(3)$ & 11.6 months & Entirely oral fed & No clinical indication for PSG to date & $\begin{array}{l}\text { Sitting with minimal support, initiating } \\
\text { rolling, able to spend time prone and } \\
\text { support head ( } 3.4 \text { months) }\end{array}$ \\
\hline $8(3)$ & 12.1 months & Entirely oral fed & Normal PSG at age 13 months & $\begin{array}{l}\text { Kicking-touches toes, stable sitting, on } \\
\text { elbows in crawling position ( } 5.4 \text { months) }\end{array}$ \\
\hline
\end{tabular}

\section{Diagnosed pre-EAP \\ SMA $1 \mathrm{C}$}

9 (3)

$10(3) \quad 5.1$ years

$11(3)$

5 years

7.9 years

$13(2) \quad 9.2$ years

$\begin{array}{ll}14(\mathrm{NA}) & 12.8 \text { years } \\ 15(3) & 14.9 \text { years }\end{array}$

16 (2) $\quad 35.7$ years
Gastrostomy and supplemental enteral feeds from age 25 months when unwell Bulbar dysfunction, NGT feeds then gastrostomy

Entirely oral fed

Entirely oral fed

Gastrostomy, enteral supplementation from age 8 years

Gastrostomy and enteral feeds $<6$ years Gastrostomy, enteral supplementation from age 5 years

NGT fed from 17 years, gastrostomy, enteral feeds from age 25 years
NIV commenced at age 25 months when unwell

NIV commenced at age 3 years, multiple prolonged PICU admissions with invasive ventilation for viral LRTIs, iatrogenic pneumothorax, HFOV

NIV commenced at age 4 years, previous Stable motor function ( 4.0 months) admission to ICU with chest infection

Normal PSG at age 8years Stable motor function (5.4 months)

Respiratory insufficiency, NIV Stable motor function (6.3 months)

commenced at age 7 years, previous PICU admissions with chest infections NIV commenced at age $<6$ years NIV commenced at age 5 years

Stable motor function (4.9 months) Stable motor function (6.3 months)

\section{Chronic respiratory insufficiency, NIV Not reported (1.8months)} commenced at age 18 years clinical setting, highlighting the management issues and practical challenges as SMA enters a new treatment era. These include difficulties in achieving early diagnosis and/or prevention, the evolution of optimal clinical care in a time of uncertain prognostication, resource implications and ethical issues.

The importance of an early diagnosis of SMA, enabling initiation of therapy at the earliest possibility, is supported by preclinical studies and data from both the nusinersen and AVXS-101 clinical trials. ${ }^{9}{ }^{10}$ Both agents are less effective in those with longer disease duration when treatment is started. Maternal child nurses, physiotherapists and paediatricians are pivotal in recognising infants presenting with hypotonia, gross motor delay and weakness, and expediting referral to neuromuscular clinics. Our patients' experiences, with marked variation in disease duration at the time of diagnosis and number of practitioners consulted, suggest that further education of healthcare professionals seeing infants at risk of SMA type 1 is necessary. Newborn screening (NBS) presents as the best opportunity to considerably reduce medical morbidity resulting from a delayed diagnosis of SMA type 1. Approximately 5\% of SMN1-related SMA cases are due to compound heterozygous SMN1 mutations, and these will not be identified with initial SMN1 gene deletion testing or current NBS approaches. Consequently it is important that typical symptomatic cases be sequenced if homozygous deletions are not present. ${ }^{11}$ Several US states have now adopted NBS for SMA. Given the availability of a treatment altering disease progression and life expectancy, we suggest that SMA should be added to the Australian NBS protocol. 


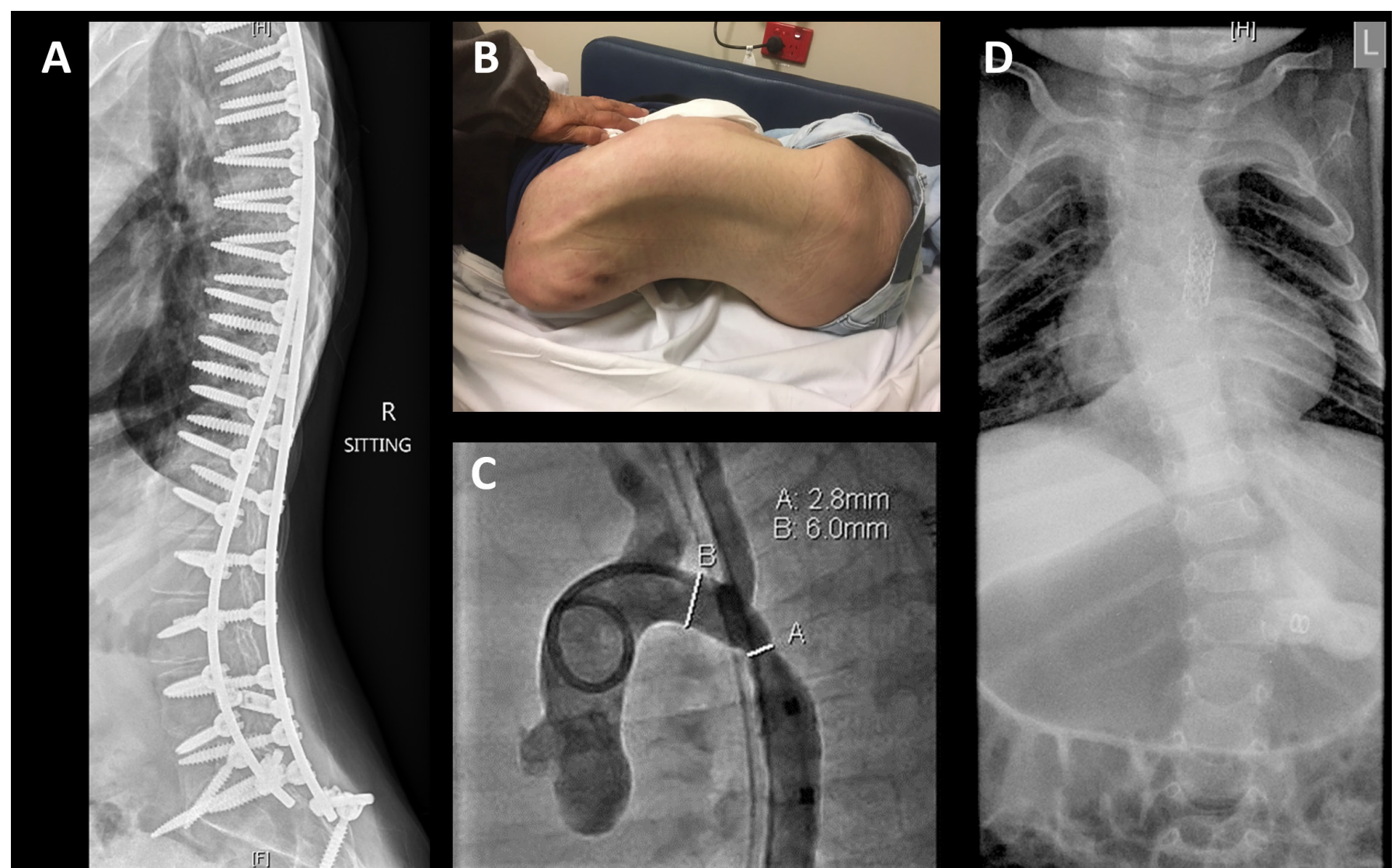

Figure 2 Unique clinical care approaches in patients with SMA type 1 treated with nusinersen. (A) Plain radiograph of spinal fusion for scoliosis necessitating CT guidance for lumbar puncture and intrathecal injection. (B) Scoliosis in adult patient with SMA type 1 pre intrathecal injection. (C) Angiogram demonstrating coarctation of the aorta in a patient with SMA type 1. (D) Chest X-ray post balloon angioplasty and placement of aortic stent on the same patient; also showing SMA type 1 comorbidities including thoracolumbar scoliosis, right upper lobe atelectasis and gastrostomy. SMA, spinal muscular atrophy.

In this series, most parents were unaware of the possibility of preconception and/or prenatal screening for SMA. These services are generally available in this country but incur a cost of $\$ 300$ $\$ 700$ and are accessed in an essentially ad hoc fashion, depending on parents' awareness, interest and ability to afford the expense of this testing. Education of health professionals, especially general practitioners, midwives and obstetricians, regarding the availability and value of preconception and prenatal screening is needed. In addition, health economic research should focus on the practicality and cost-benefits of making these tests available to all adults of childbearing age.

Australian clinicians have previously had a broad consensus regarding palliation rather than prolonged medical intervention (via tracheostomy and invasive ventilation) for infants with SMA type $1 .{ }^{12-15}$ Globally, however, there are differences regarding the level of ventilatory and nutritional support provided to patients with SMA type $1 .{ }^{16}$ We describe transformations in optimal multidisciplinary care accompanying active therapy and increased complexity of clinical decision making. As may be expected, patients diagnosed with SMA prior to initiation of the EAP were older and had the less severe SMA subtype $1 c$, in which some may survive into childhood. ${ }^{17}$ This group had already developed clinically significant bulbar and respiratory dysfunction, prompting initiation of enteral feeds and nocturnal non-invasive ventilation, on average at age 6.4 and 9.2 years, respectively. With the initiation of novel disease-modifying therapy, we have actively managed comorbidities such as undescended testes, dislocated hips and congenital heart disease. Cardiac abnormalities have previously been reported in animal models and patients with SMA type 1, but not necessarily actively looked for in a care model focused on palliation. ${ }^{718}$ Routine cardiac assessment may be indicated in patients receiving nusinersen or other disease-specific treatments for SMA type 1. Prognosis and life expectancy are now uncertain in patients treated with these novel therapies, prompting review of all aspects of supportive and palliative care. SMA type 1 remains a complex condition, and while disease outcomes are uncertain, quality of life remains a principal concern for parents, and actively managing patients with palliative care continues to be an important option.

The efficacy of the present study must be interpreted with caution, given the heterogeneous nature of this open-label cohort, which is inclusive of individuals with less severe forms of SMA type 1 and with variable disease duration. Motor function changes were measured with the HINE-2 and provide context for clinical care. The observed improvements in motor function are similar to previous reports, in which not all infants had a uniform response to nusinersen treatment. ${ }^{69}$ While the maximum and long-term response is not yet clear, at the end of the phase III randomised controlled trial on infants with symptomatic SMA type 1, 51\% achieved a motor milestone response, including head control (22\%), rolling over (10\%), sitting independently $(8 \%)$ and the ability to stand (1\%). ${ }^{9}$ Stabilisation or some improvement in motor function is considered clinically meaningful by patients and their families, ${ }^{19}$ and in this context our results suggest a shift in addressing nutritional 
and pulmonary issues earlier in newly diagnosed patients, and considering introducing proactive measures in the more severe phenotypes.

With the heightened optimism of parents, often supported by social media, there has been a new drive for intensive physiotherapy in this patient population. As this is a new clinical scenario, optimum frequency and types of physiotherapy interventions are unknown. Local physiotherapists may not have seen any children with SMA type 1 and often require support in managing affected children's complex care. Children with SMA type 1 are usually extremely weak, often requiring respiratory as well as developmental physiotherapy. Specialised equipment to ensure correct positioning is essential; poor positioning and posture may create secondary orthopaedic issues. Contractures are common in SMA type 1 and may affect motor outcome. While exercise should be encouraged, pacing is important, avoiding detrimental effects of fatigue or injury.

The reality of novel therapies, evolving natural histories and transformation of standards of care are also accompanied by clinical, logistical, financial and ethical challenges, and a number of uncertainties remain. A comprehensive understanding of the full therapeutic benefit of nusinersen will not be gained for some time, including its effects on age of death and cognitive function, need for permanent ventilation, and predictors of response. New challenges have arisen, including difficulties with access to, cost of, and resources for treatment administration (eg, the complexities and expertise required for intrathecal administration of nusinersen and supportive care). Providing ongoing supportive and drug treatment, accompanied by rapid increases in SMA prevalence, will have considerable resource implications, necessitating additional staffing and financial support. Developing novel therapies with greater efficacy and reduced burden of treatment for families and the health system is a priority. It is also vital to understand how patients and their carers make decisions regarding SMA treatment, and what aspects of treatment and quality of life are valued by patients and society in this changing treatment landscape. Taken together, these issues are critically important in promoting patient and family-centred care, enabling effective policy development, and the successful implementation of new therapies. A number of promising novel therapeutics are also in development, such that solutions to these issues are likely to be dynamic. This also has profound implications for future drug development, clinical trials and whether a predefined level of clinical care is necessary for participation in a clinical trial. ${ }^{20}$ In addition, we experienced considerable demand from parents of children with SMA types 2 and 3 who have not been offered access to nusinersen as part of the EAP, raising issues related to inequity of access. ${ }^{21}$

\section{CONCLUSIONS}

The advent of disease-modifying treatment for SMA has produced significant paradigm shifts in management and created clinical, logistical, financial and ethical challenges. It is foreseeable that similar advances will occur in other neurological disorders.

Contributors MAF designed the study and undertook data collection, analysis and interpretation, initial drafting and revisions of the manuscript, and submitted the study. HLT collected data and revised the manuscript. KAC assisted with data collection, analysis and interpretation, design of tables and figures, and revised the manuscript. AC, RF, SH, KJJ, MPM, KM, DV, IRW, RW, EMY and HS collected data and revised the manuscript. KH collected data, and assisted with drafting and revisions of the manuscript. MMR assisted with data collection, analysis and interpretation, and revised the manuscript.

Funding MAF received support from the Motor Neurone Disease Research Institute of Australia Beryl Bayley MND Postdoctoral Fellowship.

Competing interests $A C, M A F, K J J, K M, M P M, M M, M M R, H S, I R W, A C$ and DV have received honoraria from Biogen.

\section{Patient consent Obtained.}

Ethics approval Sydney Children's Hospital, Royal Children's Hospital Melbourne and Lady Cilento Children's Hospital Brisbane HRECs approved the EAP.

Provenance and peer review Not commissioned; externally peer reviewed.

Data sharing statement There are no additional unpublished data from the study.

(c) Article author(s) (or their employer(s) unless otherwise stated in the text of the article) 2018. All rights reserved. No commercial use is permitted unless otherwise expressly granted.

\section{REFERENCES}

1 Sugarman EA, Nagan $\mathrm{N}$, Zhu H, et al. Pan-ethnic carrier screening and prenatal diagnosis for spinal muscular atrophy: clinical laboratory analysis of $>72,400$ specimens. Eur J Hum Genet 2012;20:27-32.

2 Lefebvre $S$, Bürglen $\mathrm{L}$, Reboullet $\mathrm{S}$, et al. Identification and characterization of a spinal muscular atrophy-determining gene. Cell 1995;80:155-65.

3 Wang $\mathrm{CH}$, Finkel RS, Bertini ES, et al. Consensus statement for standard of care in spinal muscular atrophy. J Child Neurol 2007;22:1027-49.

4 Chiriboga CA, Swoboda KJ, Darras BT, et al. Results from a phase 1 study of nusinersen (ISIS-SMN(Rx)) in children with spinal muscular atrophy. Neurology 2016;86:890-7.

5 Haché M, Swoboda KJ, Sethna N, et al. Intrathecal Injections in Children With Spinal Muscular Atrophy: Nusinersen Clinical Trial Experience. J Child Neurol 2016:31:899-906.

6 Finkel RS, Chiriboga CA, Vajsar J, et al. Treatment of infantile-onset spinal muscular atrophy with nusinersen: a phase 2, open-label, dose-escalation study. Lancet 2016;388:3017-26.

7 Farrar MA, Park SB, Vucic S, et al. Emerging therapies and challenges in spinal muscular atrophy. Ann Neurol 2017;81:355-68.

8 Frisone MF, Mercuri E, Laroche $S$, et al. Prognostic value of the neurologic optimality score at 9 and 18 months in preterm infants born before 31 weeks' gestation. J Pediatr 2002;140:57-60.

9 Finkel RS, Mercuri E, Darras BT, et al. Nusinersen versus sham control in infantileonset spinal muscular atrophy. N Engl J Med 2017;377:1723-32.

10 Mendell JR, Al-Zaidy S, Shell R, et al. Single-dose gene-replacement therapy for spinal muscular atrophy. N Engl J Med 2017;377:1713-22.

11 Farrar MA, Johnston HM, Grattan-Smith P, et al. Spinal muscular atrophy: molecular mechanisms. Curr Mol Med 2009;9:851-62.

12 Geevasinga N, Ryan MM. Physician attitudes towards ventilatory support for spinal muscular atrophy type 1 in Australasia. J Paediatr Child Health 2007;43:790-4.

13 Ryan MM. The use of invasive ventilation is appropriate in children with genetically proven spinal muscular atrophy type 1: the motion against. Paediatr Respir Rev 2008;9:51-4.

14 Tassie B, Isaacs D, Kilham $\mathrm{H}$, et al. Management of children with spinal muscular atrophy type 1 in Australia. J Paediatr Child Health 2013;49:815-9.

15 Farrar MA, Vucic S, Johnston HM, et al. Pathophysiological insights derived by natural history and motor function of spinal muscular atrophy. J Pediatr 2013;162:155-9.

16 Bladen CL, Thompson R, Jackson JM, et al. Mapping the differences in care for 5,000 spinal muscular atrophy patients, a survey of 24 national registries in North America, Australasia and Europe. J Neurol 2014;261:152-63.

17 Wadman RI, Stam M, Gijzen M, et al. Association of motor milestones, SMN2 copy and outcome in spinal muscular atrophy types 0-4. J Neurol Neurosurg Psychiatry 2017;88:365-7.

18 Wijngaarde CA, Blank AC, Stam M, et al. Cardiac pathology in spinal muscular atrophy: a systematic review. Orphanet J Rare Dis 2017;12:67.

19 Pera MC, Coratti G, Forcina N, et al. Content validity and clinical meaningfulness of the HFMSE in spinal muscular atrophy. BMC Neurol 2017;17:39.

20 Finkel RS, Bishop KM, Nelson RM. Spinal muscular atrophy type I: is it ethical to standardize supportive care intervention in clinical trials? J Child Neurol 2017;32:155-60

21 Burgart AM, Magnus D, Tabor HK, et al. Ethical challenges confronted when providing nusinersen treatment for spinal muscular atrophy. JAMA Pediatr 2018;172:188-92. 